\title{
Study and Analysis of the Filter Clogging Effect on the Pressure Losses of an Axial Fan
}

\author{
Azzedine BOUZAOUIT \\ University of Skikda, Algeria, bouzaouit21@gmail.com \\ Mounir GAHGAH \\ University of Skikda, Algeria, gahgah.mounir@gmail.com \\ Mouna LAIFA \\ University of Skikda, Algeria, mouna.laifa@hotmail.com \\ Leila RAMECHE \\ University of Skikda, Algeria, leila.rameche@outlook.com
}

\begin{abstract}
We present in this paper the results of an experimental study conducted on the analysis of the clogging influence of a filter installed on the suction pipe, on the axial fan operating parameters. In this study, we used an axial fan driven by a variable speed electric motor. The filter used has a removable filter element, in this context, we tested five filter elements with different filter surfaces and this to model the effect of clogging the filter on the pressure losses generated at the level of the filter in question. To obtain a differential pressure gauge installed upstream and downstream of the filter is used, the pressure value detected by the latter characterizes the relationship between the filtering surface (the surface crossed by the air flow at the filter) and the singular losses. The tests were carried out for different flow rates and speeds. The results obtained clearly show the effect of the filter clogging on the operating parameters of the fan, and characterize the relationship of these parameters with local pressure losses.
\end{abstract}

\section{Keywords}

fluid flow, suction filter, clogging, local pressure drops, axial fan

\section{Introduction}

Fluid flow is a complex phenomenon. We are interested in the fundamental equations that govern the dynamics of real incompressible fluids, in particular:

- The continuity equation (conservation of the mass),

- Bernoulli's theorem (conservation of energy),

- Euler's theorem (conservation of the momentum) from which the equations giving the dynamic force exerted by the moving fluids are established [1].

Experimentally, two fluid flow regimes (laminar or turbulent) can be easily distinguished. The fluid flow regime, in the case of a forced flow provided by a pressure machine such as pump, fan ..., varies according to the technical characteristics of each machine. If it is, an axial fan that creates an air flow that runs parallel to its axis of rotation. The essential characteristics defining a fan are manometry height or total pressure, efficiency, flow and the rotation speed. The absorbed power of an axial fan is one of its most desirable characteristics, it describes the amount of energy required to operate the device [2].

When the air moves in a pipe under the effect of the fan mechanical action between two points (1) and (2) located on the suction or discharge pipe, it undergoes losses due to friction on the walls of the pipes, in the elbows more or less tight and in all other obstacles, he will encounter in his path.

The pressure drop is considered a loss of energy and consequently a reduction in the efficiency; it must be compensated to allow the fluid to move. It is commonly expressed as a pressure difference $(\Delta \mathrm{P})$, although it is actually representative of energy dissipation and appears in the Bernoulli equation:

$$
\frac{1}{2} \cdot \rho \cdot v_{1}^{2}+\rho \cdot g \cdot z_{1}+p_{1}=\frac{1}{2} \cdot \rho \cdot v_{2}^{2}+\rho \cdot g \cdot z_{2}+p_{2}+\Delta P_{t}
$$

with: $p_{1}, p_{2}$ : the pressures in points 1 and 2 ; 
$\rho$ : the fluid density;

$v_{1}, v_{2}$ : the flow velocities of the fluid in points 1 and 2 ;

$g$ : the acceleration of gravity;

$Z_{1}, z_{2}$ : altitude at points 1 and 2 ;

$\Delta P_{t}$ : total pressure loss,

$$
\Delta P_{t}=\Delta P_{L}+\Delta P_{S}
$$

There are two types of pressure loss:

The systematic or linear pressure loss $\left(\Delta P_{L}\right)$ results from the friction exerted between the fluid and the inner surface of the pipe [3]. They are proportional to the pipe length $L$ and the square of the fluid average flow velocity $V$; they are inversely proportional to the diameter $D$ and function of the average roughness $\varepsilon$ of the pipe. Between two separate points along a length $L$, in a pipe of diameter $D$ appears a pressure loss $\Delta P_{L}$ given by the following Darcy equation:

$$
\Delta P_{L}=\frac{\varepsilon}{D} \cdot \frac{\rho \cdot V^{2}}{2} \cdot L .
$$

The singular pressure losses $\left(\Delta P_{S}\right)$ result from the presence of bends $[4,5,6]$, connection, tap, installation of a filter, valves. All these elements (singularities) installed along the pipe, constitute obstacles which slow down the free passage of air and bring about local pressure losses proportional to the square of the flow velocity, they are given by equation (4):

$$
\Delta P_{S}=K \cdot \frac{\rho \cdot V^{2}}{2},
$$

with: $\rho$ : the fluid density,

$V$ : flow velocity,

$K$ : coefficient depending on the nature of the local resistance (module load loss).

In a ventilation or air extraction system with the use of an axial-type fan, the existence of a filter on the suction line to provide protection against contamination of undesirable particles in general, as well as preserving the elements of the circuit and the environment at the same time is important [7]. Filters are often used in all ventilation systems.

Monitoring the fan behavior in order to analyze the clogging effect of a filter installed on the suction pipe and to characterize its influence on the machines operating parameters, taking into account the evolution of singular pressure losses at the filter, this is the objective of our work.

\section{Methodology of Work}

The objective of the present work is to evaluate and characterize the clogging effect of a filter installed on the suction pipe of a forced air system on local pressure losses. For this, we used an experimental setup; it is given by the Figure 1 to perform the tests necessary to achieve our objective.

The experimental device consists mainly of an axial fan with variable drive speed, on the suction line is installed a coarse filter with removable filter element, in order to modify its filtering surface, in this context, we used five filter elements each with a specific clogging rate.

A pressure sensor $\Delta \mathrm{P} 1$ is installed on both sides of the filter to measure the pressure difference generated at the filter level. Another $\Delta \mathrm{P} 2$ is installed at the fan to measure the pressure difference between the output and the input, in order to deduce the power of the fan if it is needed.

The pressures detected at the considered measurement points will be stored in a data collector for display on a PC for possible processing and analysis.

\section{Results and Discussion}

After commissioning of the experimental device, we tested system without filter element at different speeds whose values in (Hz) are respectively: 10.40; 20.64; 30.40; 40.16 and 44.38, then we used the five elements separately, the different values of the local pressure losses obtained are given in Table 1 and Table 2 . 


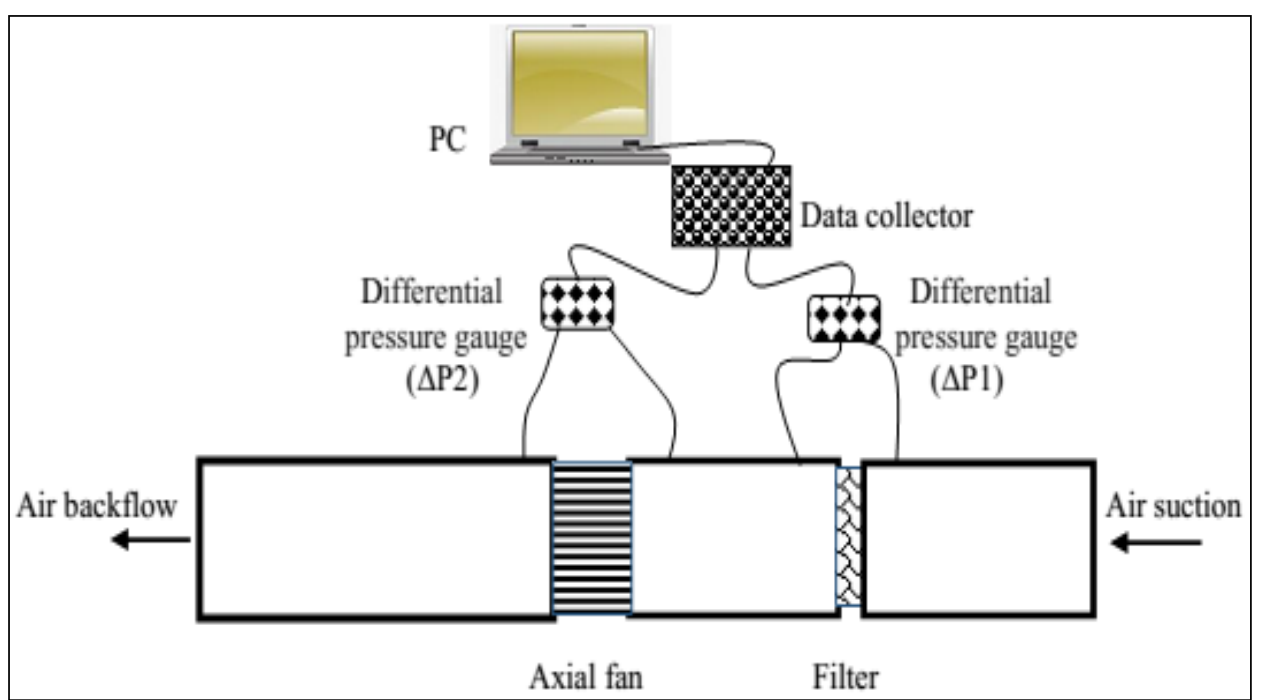

Fig. 1. The experimental device

Table 1. Experimental values of pressure losses

\begin{tabular}{|c|c|l|l|l|l|l|}
\hline Clogging filter & \multirow{2}{*}{ Withoutfilter } & \multicolumn{1}{|c|}{0} & $25 \%$ & $50 \%$ & $75 \%$ & $95 \%$ \\
\hline $\mathrm{N} 1=10.40$ & 0.81 & 1.01 & 1.18 & 1.28 & 1.49 & 1.75 \\
\hline $\mathrm{N} 2=20.64$ & 0.9 & 1.8 & 2.6 & 2.7 & 3.4 & 4.0 \\
\hline $\mathrm{N} 3=30.40$ & 2.0 & 2.33 & 2.58 & 2.85 & 3.34 & 3.66 \\
\hline $\mathrm{N} 4=40.16$ & 3.56 & 3.98 & 4.39 & 4.94 & 5.58 & 6.42 \\
\hline $\mathrm{N} 5=44.38$ & 4.57 & 4.65 & 5.16 & 5.65 & 6.25 & 6.77 \\
\hline
\end{tabular}

Table 2. Pressure loss as function of flow (for $\mathrm{N}=44.38 \mathrm{~Hz}$ )

\begin{tabular}{|c|c|c|c|c|c|c|}
\hline$Q\left(\mathrm{~m}^{3} / \mathrm{s}\right)$ & 0.0403 & 0.0411 & 0.0432 & 0.0451 & 0.0473 & 0.0495 \\
\hline$\Delta \mathrm{P} 1(\mathrm{~mm} . \mathrm{Wc})$ & 4.57 & 4.65 & 5.16 & 5.65 & 6.25 & 6.77 \\
\hline
\end{tabular}

The evolution of the filter element clogging has direct and indirect effects on the machine performance parameters, these effects have been characterized by variable local pressure losses $(\Delta \mathrm{P})$ produced by the change of the state of the filter element used, in other words the filter clogging, they are presented by Figure 2 .

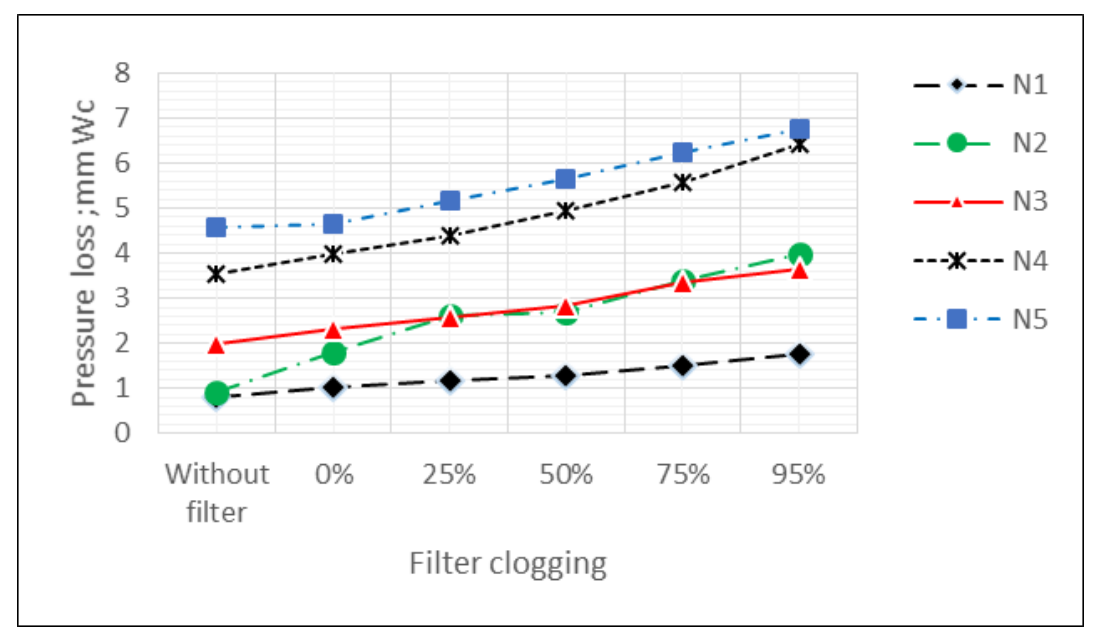

Fig. 2. Pressure losses depending on the clogging state of filter 
The operating parameters of the fan such as the flow rate, the rotational speed (for example: $\mathrm{N}=44.38 \mathrm{~Hz}$ ) have a significant influence on the pressure losses in particular and on the overall efficiency of the machine, this influence is represented by the Figure 3.

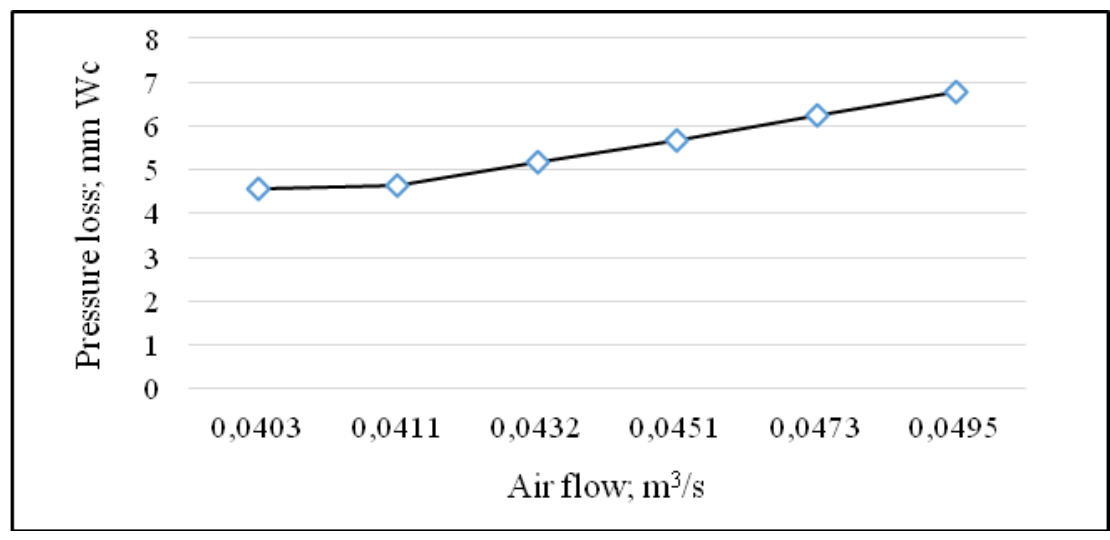

Fig. 3. Pressure losses depending on the fan flow

In the same way, knowing that the air flow is a function of the rotation speed of the fan, so the pressure losses in the circuit are also related to the driving speed of the machine, this characteristic is given by the Figure 4 .

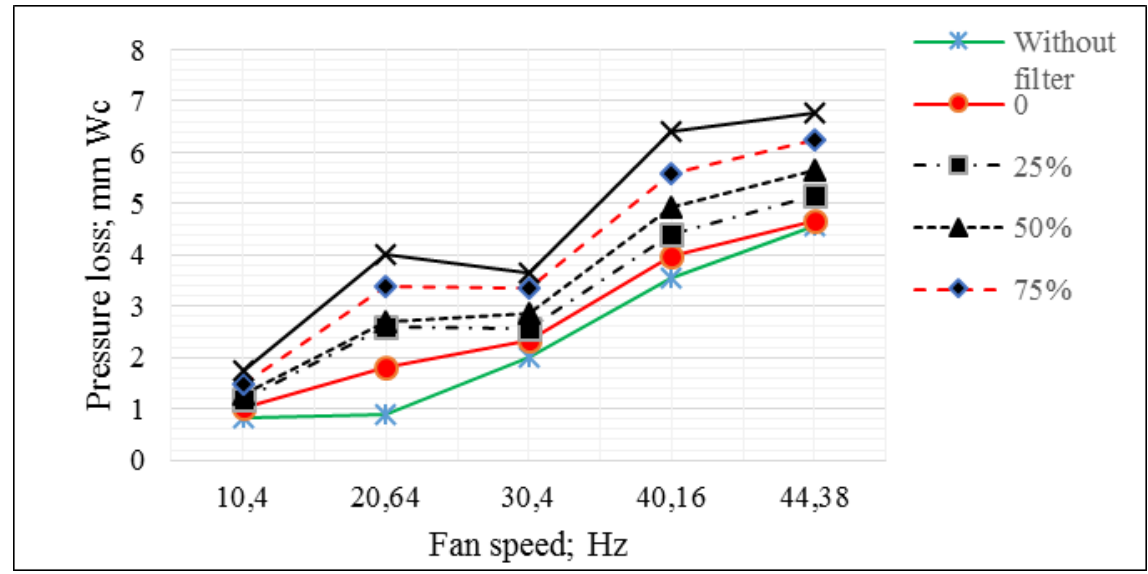

Fig. 4. Pressure losses depending on the rotation speed of the fan

From the Figure 2, it is clear that the pressure loss $(\Delta \mathrm{P})$ increases with the degradation of the filter element state. So to preserve the filter used and to make it exploit in proper conditions, that is to say by respecting the value of the coefficient of allowable singular pressure losses, in order to maintain a better overall efficiency of the fan, a preventive maintenance program based essentially on the cleaning of the filter element must be instituted, with a periodicity that depends on the concentration of the polluting particles.

In the same way, the Figure 4 shows that the pressure losses $(\Delta \mathrm{P})$ are very sensitive to the variation of the fan rotational speed; they increase with the increase of the latter.

Knowing that the flow rate of the sucked air is related to the rotation speed, the pressure losses also increase with the increase of the flow rate air, it is given by Figure 3 .

\section{Conclusion}

The air flow between two areas is mainly due to the difference of pressure between the locations considered. The air flow through such a system requires energy to overcome the static pressure at the inlet or the outlet of the system. The fan provides this energy because of the variation of the total pressure between the inlet and the outlet. 
Therefore, fans in general are devices of fluids compression; they are intended in addition to guarantee the safety to preserve the people's health evolving in certain professional circles and to answer industrial processes, ensuring the extraction of polluted air in the work areas. The role of fans is paramount in the field of industrial activity, they provide a very suitable environment to work.

To do this, the use of filters in ventilation systems to provide protection against contamination that can lead to malfunctions and damage to the industrial system is necessary. The pressure of the circulating air decreases all along a pipe in which it flows, and even when passing through an obstacle, such as elbow, valve, shrink or filter, it is called local or singular pressure losses.

The present study is devoted to an experimental characterization of the parameters of an axial fan, under the influence of the pressure losses, caused by the clogging of the polluting particles on the filter installed on the suction pipe. The latter is a means of protection against contamination that can lead to malfunction and damage to the system.

We have proved through this study, which the loss on the stored energy of a fluid is in direct relation with the operating conditions of the fan used such as; the rotation speed, the flow rate and even the state of air conveyed pollution.

The fan efficiency is very sensitive to the evolution of the filter clogging by the polluting particles. A preventive maintenance program is considered necessary for the filter cleaning in order to guarantee the machine performance and to preserve the health of the operators.

\section{References}

1. **: http://fsa.univ-tiaret.dz/departements/st/formations/fichierjoint/cours/mdf.pdf. Accessed: 2015

2. ***: https://www.thermexcel.com/french/ressourc/dimensionnement_ventilateur_ventilateurs_pression.htm. Accessed: 2017

3. Barabas B., Deaconescu T. (2016): Hydroabrasive machining process optimization through statistical analysis and robust design. RECENT, ISSN: 1582-0246, Vol. 17, no. 4(50), p. 502-506

4. Adam N.J., De Cesare G., Schleiss A.J., Richard S., Muench-Alligné C. (2016): Head loss coefficient through sharpedged orifices. 28th IAHR Symposium on Hydraulic Machinery and Systems, July 4th-8th 2016, Grenoble, France. Published in IOP Conf. Ser.: Earth Environ. Sci. 49 062009, eISSN: 1755-1315, p. 205-213

5. Zardin B., Cillo G., Rinaldini C.A., Mattarelli E., Borghi M. (2017): Pressure losses in hydraulic manifolds. Energies, ISSN: 1996-1073, Vol. 10(3), 310, p. 03-21, https://doi.org/10.3390/en10030310

6. Bouzaouit A., Hadjadj A., Gahgah M., Bennis 0. (2009): Pollution hydraulic fluids analysis by the differential pressure filters evolution. U.P.B. Sci. Bull., Series D, ISSN: 1454-2358, Vol. 71, Iss.4, p. 183-190

7. ***: http://www.ventilation-alnor.fr/index/support/base-du-savoir/ventilation-FR. Accessed: 2013-12-04 